\title{
Study on the Application of Systemic Inflammation Response Index and Platelet-Lymphocyte Ratio in Ovarian Malignant Tumors
}

\author{
Huifang Huang' \\ Kunhai $\mathrm{Wu}^{2}$ \\ Lufei Chen ${ }^{2}$ \\ Xiaomei Lin'
}

'Intensive Care Unit, Fujian Maternity and Child Health Hospital, Affiliated Hospital of Fujian Medical University, Fuzhou, Fujian, People's Republic of China; ${ }^{2}$ Blood Transfusion Department, Fujian Maternity and Child Health Hospital, Affiliated Hospital of Fujian Medical University, Fuzhou, Fujian, People's Republic of China
Correspondence: Kunhai Wu; Lufei Chen Tel +86 I390692/205; +86 I3705032570 Fax +86 59I-87555474

Email 315084702@qq.Com; 502108678@qq.Com
Objective: We aimed to evaluate whether the systemic inflammatory response index (SIRI) and platelet lymphocyte ratio (PLR) are associated with ovarian malignancy and their diagnostic value. Design: This retrospective study compared SIRI, PLR, cancer antigen 125 (CA125), cancer antigen 153 (CA153), cancer antigen 199 (CA199), A carcinoma embryonic antigen (CEA) and alpha fetal protein (AFP) of the two groups in Fujian Maternity and Child Health Hospital, Affiliated Hospital of Fujian Medical University from January 2018 to December 2020, divided into two groups based on pathological results, including 85 with ovarian malignancy who met the study criteria and 85 patients with benign ovarian tumors were randomly selected as control group.

Results: 1) SIRI and PLR in benign ovarian tumor group were lower than those in ovarian malignancy group; 2) SIRI and PLR in ovarian malignant tumor low stage group were lower than those in the high stage group; 3 ) In ovarian malignancies, SIRI and PLR were positively associated with CA125 (the correlation coefficient $r=0.251, p=0.021 ; r=0.251, p=0.020$;) but showed no correlation with CA153.

Conclusion: The study shows that SIRI and PLR are both convenient and associated with ovarian malignancy. SIRI and PLR can be used to help the differentiation of benign and malignant ovarian tumors and can also be used in the markers of ovarian malignant tumors roughly staging.

Keywords: ovarian malignancy, SIRI, PLR

\section{Introduction}

Ovarian cancer is one of the most common tumors in worldwide. It can be divided into ovarian malignant tumors and ovarian benign tumors. Ovarian malignant tumor is the most fatal gynecologic malignancy, ${ }^{1}$ and the onset is only second to cervical cancer and endometrial cancer. ${ }^{2}$ Although ovarian cancer in early stage can be successfully treated, it is commonly detected at advanced stages with extensive local and systemic spread and poor survival. The 5-year survival rate of patients with ovarian malignant tumor stage I is $90 \%$, while patients with stage IV is insufficient $11 \%{ }^{3}$ Therefore, early diagnosis of ovarian malignant tumors may effectively reduce mortality.

Diagnosis of ovarian malignant tumors is based on history, physical examination, tumor biomarker tests, imaging examination and histopathological examination. However, these inspections take much time and are expensive. The asymptomatic nature of ovarian cancer makes it difficult to detect in the early 
stages. ${ }^{4}$ The study found that CA125 lacks specificity in the clinical diagnosis of ovarian cancer, ${ }^{5}$ CA153 was first discovered in breast cancer epithelial cells. CA19-9 has good specificity for the diagnosis of gastrointestinal tumors, and has a synergistic effect on the diagnosis of ovarian cancer. ${ }^{6,7}$ AFP is of great significance to the diagnosis and evaluation of ovarian cancer. ${ }^{8} \mathrm{Up}$ to $50 \%$ of patients with epithelial ovarian cancer have elevated CEA levels. ${ }^{9}$ The tumor biomarker did not significantly changed in some early ovarian malignant tumor. Therefore, we need easier tests to screen for ovarian malignancies.

Multiple lines of evidence suggest that ovarian cancer may be related to chronic inflammation. ${ }^{10}$ SIRI and other indicators related to inflammation are closely related to the recurrence and overall survival of many malignant tumors. NLR, MLR, and PLR are good indicators to reflect systemic inflammation. ${ }^{11}$ More and more studies have found that there is a significant correlation between malignant tumors and PLR. Some studies have confirmed that platelets play an important role in the growth, metastasis and angiogenesis of malignant tumors. ${ }^{12}$ This paper focuses on the correlation of SIRI and PLR with ovarian tumors, in order to provide a reference index for the clinical screen of ovarian tumors.

\section{Materials and Methods Study Population and Data}

This was a retrospective study, including women who were admitted to Fujian Maternity and Child Health Hospital, Affiliated Hospital of Fujian Medical University for surgical treatment from January 2018 to December 2020. SIRI is the ratio of neutrophil $\times$ monocyte/lymphocyte; PLR is the ratio of platelet to lymphocyte.

Inclusion criteria included: a. new patients who were diagnosed with benign and malignant ovarian tumors and will undergo surgery; $b$. confirmed by postoperative pathology; c. detailed case information. Exclusion criteria included: a. combined with cardiovascular disease, diabetes, autoimmune disease, blood system disease; b. combined with other site tumor or tumor metastasis; c. treatment such as radiotherapy and chemotherapy; d. severe infection; e. anticoagulant treatment (oral anticoagulants, acetylsalicylic acid, statins, etc.)

There were 170 ovarian malignancy women, and 85 patients met the inclusion criteria (age range 19-66 years old, average age $49.00 \pm 9.391$ years). 85 patients with benign ovarian tumors of the same period were randomly selected as the control group (age range 18-65 years old, average age $48.06 \pm 10.471$ years old). There was no statistically significant difference in the age of the two groups, and they were comparable.

\section{Staging of Ovarian Malignancy}

All patients with ovarian malignant tumors are staged according to FIGO 2014. They were divided into two groups: stage I-II is the low-stage ovarian cancer group, and stage III-IV is the high-stage ovarian cancer group.

\section{Study Variables}

Fasting venous blood from all patients with ovarian tumors was drawn in the morning within one week before surgery for routine blood tests and tumor marker detection. Fasting refers to fasting for more than 8 hours the night before. The blood routine uses an automatic blood analyzer to count the neutrophil count, monocyte count, lymphocyte count, and platelet count. Calculate the SIRI and PLR values based on the numerical values. We used Sysmex CS-5100 as the testing instrument. The biochemical tests (include CA125, CA153, CA199, CEA, AFP, etc.) used the Abbott al16200 (Abbott, Chicago, USA) as the testing instrument.

\section{Statistical Methods}

Use SPSS24.0 statistical software to compare and analyze the data. Continuous variables were presented as mean \pm standard deviation (SD) and compared by $t$-test, ANOVA and Wilcoxon rank-sum test depending on their distribution. Application of receiver operating characteristic curve (ROC curve) was used to evaluate the diagnostic value of SIRI and PLR. P-values less than 0.05 were considered statistically significant. Significance was set at $\mathrm{P} \leq 0.05$. Correlation analysis adopts PEARSON analysis.

\section{Results \\ Comparison of Clinical Indicators Between Ovarian Malignant Tumors and Ovarian Benign Tumors}

SIRI and PLR both had significant differences between the two groups, the ovarian malignant tumor group was significantly higher than the ovarian benign tumor group, the differences were statistically significant $(\mathrm{P}<0.001)$; These results were presented in Table 1. 
Table I Comparison of Clinical Indicators Between Ovarian Malignant Tumors and Ovarian Benign Tumors

\begin{tabular}{|l|c|c|c|c|}
\hline & $\begin{array}{c}\text { Ovarian Malignant Tumors } \\
(\mathbf{n = 8 5})\end{array}$ & $\begin{array}{c}\text { Ovarian Benign Tumors } \\
(\mathbf{n = 8 5})\end{array}$ & $\mathbf{t}$ \\
\hline Age(y) & $49.00 \pm 9.391$ & $48.06 \pm 10.471$ & 0.541 & $>0.05$ \\
SIRI & $1.46 \pm 0.97$ & $0.71 \pm 0.36$ & 6.56 & $<0.001$ \\
PLR & $177.12 \pm 68.58$ & $138.84 \pm 40.44$ & 4.101 & $<0.001$ \\
\hline
\end{tabular}

Notes: $P<0.05$ indicates statistical significance, $P>0.05$ indicates no statistical significance.

Abbreviations: SIRI, systemic inflammatory response index; PLR, platelet lymphocyte ratio.

\section{The Association Between SIRI and PLR with the Ovarian Malignant Tumor}

The AUC for each factor was as follows: SIRI level, 0.793 (95\% CI 0.727 to $0.859 ; \mathrm{p}<0.001$ ), with SIRI above 0.69 yielded a sensitivity of $82.4 \%$ and specificity of $62.7 \%$ (Figure 1A); PLR level, 0.667 (95\% CI 0.586 to 0.747; $\mathrm{p}<0.001$ ), with PLR above 157.68 provided with a sensitivity of $55.3 \%$ and specificity of $72.9 \%$ (Figure 1B); The ROC curve analysis showed that SIRI and PLR were useful indicators of predicting benign and malignant ovarian tumors before surgery.

\section{Comparison of the Clinical Indicators in the Different Stages of the Ovarian \\ Malignant Tumor Group}

All patients with ovarian malignant tumors are staged according to FIGO 2014. They were divided into two

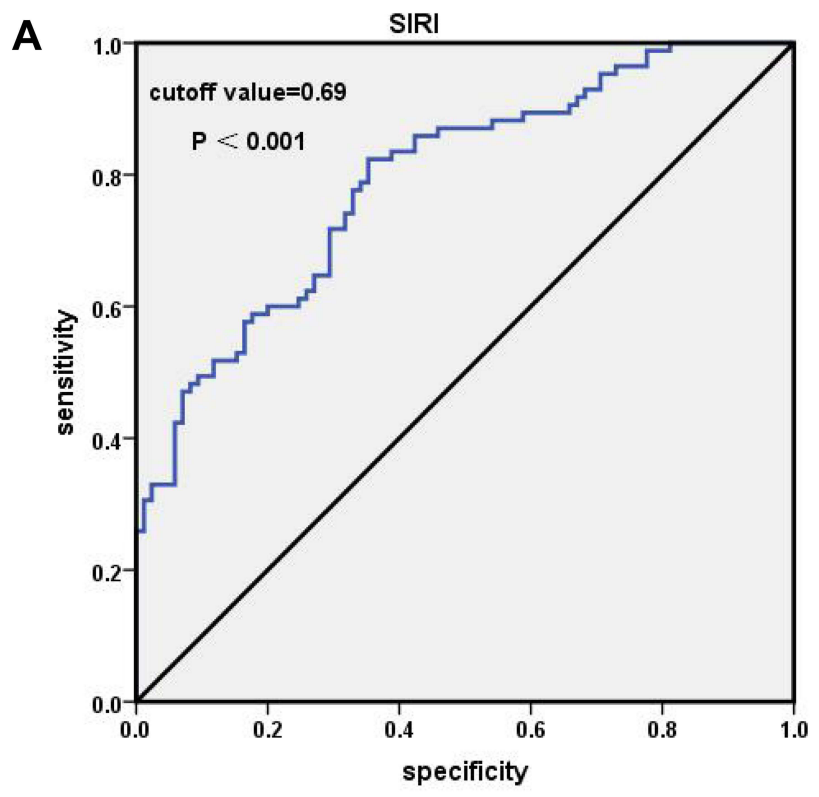

groups: stage I-II is the low-stage ovarian cancer group, and stage III-IV is the high-stage ovarian cancer group.

The levels of CA199, CEA and AFP had no significant differences between the groups. In the high-stage group as compared to the low-stage group, there were higher SIRI (1.81 vs $1.25, \mathrm{p}<0.05)$, higher PLR (204.84 vs 160.38 , $\mathrm{p}<0.05)$, higher CA125 $(\mathrm{U} / \mathrm{mL})(80.70$ vs 44.26 , $\mathrm{p}<$ $0.001)$ and CA153 (U/mL) $(29.18$ vs $14.13, \mathrm{p}<0.001)$. (Table 2).

\section{The Association Between Variables with the Different Stages of the Ovarian}

\section{Malignant Tumor}

For factors that were significantly different, the ROC curves were obtained to define the optimal cut-off values. The AUC for each factor was as follows: SIRI level, 0.682 (95\% CI 0.566 to $0.798 ; \mathrm{p}=0.005$ ), with SIRI above 1.21

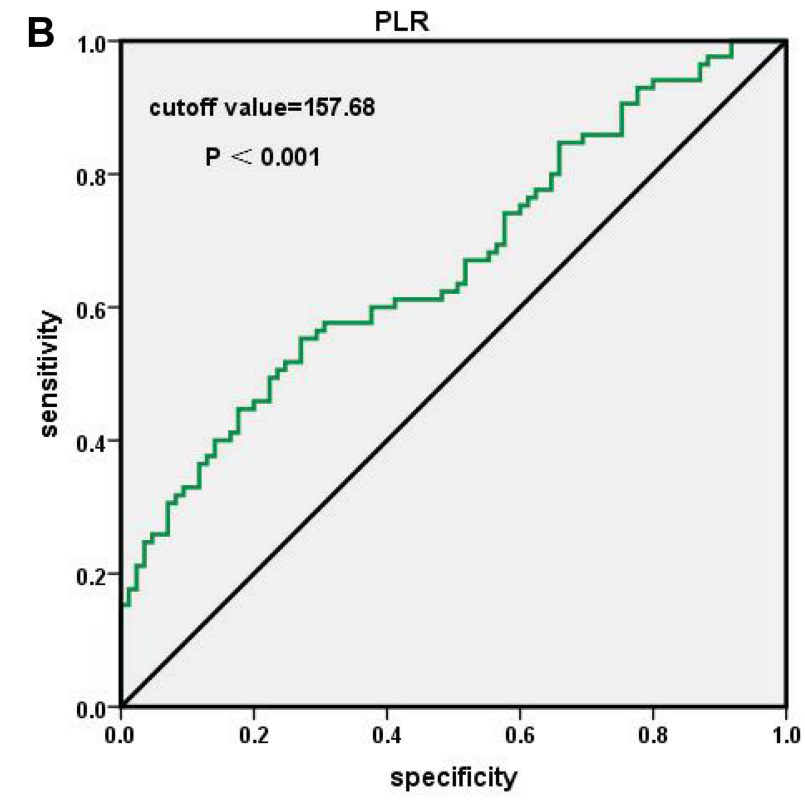

Figure I (A) ROC curve analysis for the association between SIRI with the ovarian malignant tumor. (B) ROC curve analysis for the association between PLR with the ovarian malignant tumor.

Note: $\mathrm{P}<0.00$ I suggests significantly different.

Abbreviations: SIRI, systemic inflammatory response index; PLR, platelet lymphocyte ratio. 
Table 2 Comparison of the Clinical Indicators in the Different Stages of the Ovarian Malignant Tumor Group

\begin{tabular}{|l|c|c|c|c|}
\hline & $\begin{array}{c}\text { I-II } \\
\mathbf{( N = 5 3 )}\end{array}$ & $\begin{array}{c}\text { III-IV } \\
\mathbf{( N = 3 2 )}\end{array}$ & $\mathbf{t}$ \\
\hline SIRI & $1.25 \pm 0.80$ & $1.81 \pm 1.12$ & -2.675 & $<0.05$ \\
PLR & $160.39 \pm 63.05$ & $204.84 \pm 69.32$ & -3.033 & $<0.05$ \\
CAI25(U/mL) & $44.26 \pm 39.31$ & $80.70 \pm 47.74$ & -3.817 & $<0.001$ \\
CAI53(U/mL) & $14.13 \pm 10.74$ & $29.18 \pm 18.93$ & -4.681 & $<0.001$ \\
CAI99(U/mL) & $10.17 \pm 6.69$ & $11.24 \pm 8.40$ & -0.649 & $>0.05$ \\
CEA $(\mathrm{ng} / \mathrm{mL})$ & $2.10 \pm 1.47$ & $1.95 \pm 1.00$ & -0.526 & $>0.05$ \\
AFP(ng/mL) & $2.50 \pm 1.56$ & $2.69 \pm 1.18$ & -0.583 & $>0.05$ \\
\hline
\end{tabular}

Notes: $P<0.05$ indicates statistical significance, $P>0.05$ indicates no statistical significance.

Abbreviations: SIRI, systemic inflammatory response index; PLR, platelet lymphocyte ratio; CAI25, cancer antigen I25; CAI53, cancer antigen I53; CAI99, cancer antigen 199; CEA, A carcinoma embryonic antigen; AFP, alpha fetal protein.

yielded a sensitivity of $71.9 \%$ and specificity of $62.3 \%$ (Figure 2A); PLR level, 0.708 (95\% CI 0.592 to 0.823 ; $\mathrm{p}=0.01$ ), with PLR above 182.0 provided with a sensitivity of $65.6 \%$ and specificity of $71.7 \%$ (Figure $2 \mathrm{~B}$ ).

The AUC for CA125 level was $0.782(95 \%$ CI 0.685 to $0.879 ; \mathrm{p}<0.001)$ and a cutoff point $\geq 40.93 \mathrm{U} / \mathrm{mL}$ characterized by a sensitivity of $84.4 \%$ and a specificity of 69.8\%; The AUC for CA153 level was 0.821 (95\% CI 0.730 to $0.912 ; \mathrm{p}<0.001)$ and a cutoff point $\geq 12.7 \mathrm{U} / \mathrm{mL}$ characterized by a sensitivity of $93.8 \%$ and a specificity of $64.2 \%$ (Figure 2C); The ROC curve analysis showed that SIRI and PLR were significantly associated with the development of the high-stage ovarian malignant tumor as CA125 and CA153.

\section{Comparison of SIRI, PLR Correlation with CAI25, CAI53 in Ovarian \\ Malignancies}

In ovarian malignant tumors, SIRI was positively correlated with CA125, the correlation coefficient was $r=0.251$, $\mathrm{p}=0.021$; PLR was positively correlated with CA125, the correlation coefficient was $\mathrm{r}=0.251, \mathrm{p}=0.020$; SIRI was not correlated with CA153 ( $\mathrm{P}>0.05)$; PLR was not correlation with CA153 $(\mathrm{P}>0.05)($ Table 3, Figure $3 \mathrm{~A}$ and $\mathrm{B})$.

\section{Discussion}

To our knowledge, this is the first study associated SIRI, PLR and ovarian tumors. Our study showed that SIRI and PLR levels were significantly different between patients with ovarian benign tumors and patients with ovarian malignant tumors. The ROC curves showed that SIRI and PLR levels could be used in the preoperative prediction of benign and malignant ovarian tumors, and might also be used to predict the different stages of ovarian malignant tumors.
As we know, the incidence of ovarian malignant tumors ranks third in female reproductive system tumors, second only to cervical cancer and intrauterine cancer. The mortality of ovarian malignant tumors is much higher than the other two. The main reasons are that early diagnosis of ovarian malignant tumors is difficult, the recurrence rate is high after surgery, and longterm chemotherapy leads to drug resistance in cancer cells. $^{13}$

At present, the clinical diagnosis methods of ovarian tumors mainly include histopathological examination, imaging examination and markers examination. Histopathological examination, as the "gold standard" of diagnosis, ${ }^{14}$ is an invasive inspection and not suitable as the first-choice examination. Imaging examination can understand tumor location, size, depth of invasion, invasion range, lymph node condition, etc, but it is not easy to find smaller lesions; Moreover, examinations such as MRI, $\mathrm{CT}$, and PET-CT are expensive, and also not suitable as routine examination items.

Serological examination mainly includes the detection of tumor markers such as CA125 and human epididymal secretory protein 4 (HE4). CA125 is a mucinous glycoprotein, often used in the diagnosis of epithelial ovarian malignancies. However, there are many factors affecting the increase of CA125. Endometriosis, endometrial cancer, gastrointestinal tumors etc., can all lead to the increase of CA125, and it is not suitable to be used alone as a diagnostic indicator for ovarian malignant tumors. It has been reported in the literature that CA125 is not sensitive in the early stage of ovarian malignant tumors, and only $23-50 \%$ of patients with elevated CA125 in stage I cases. ${ }^{15}$ At the end of the 20th century, researchers discovered that HE4 can be expressed in $93 \%$ of serous 

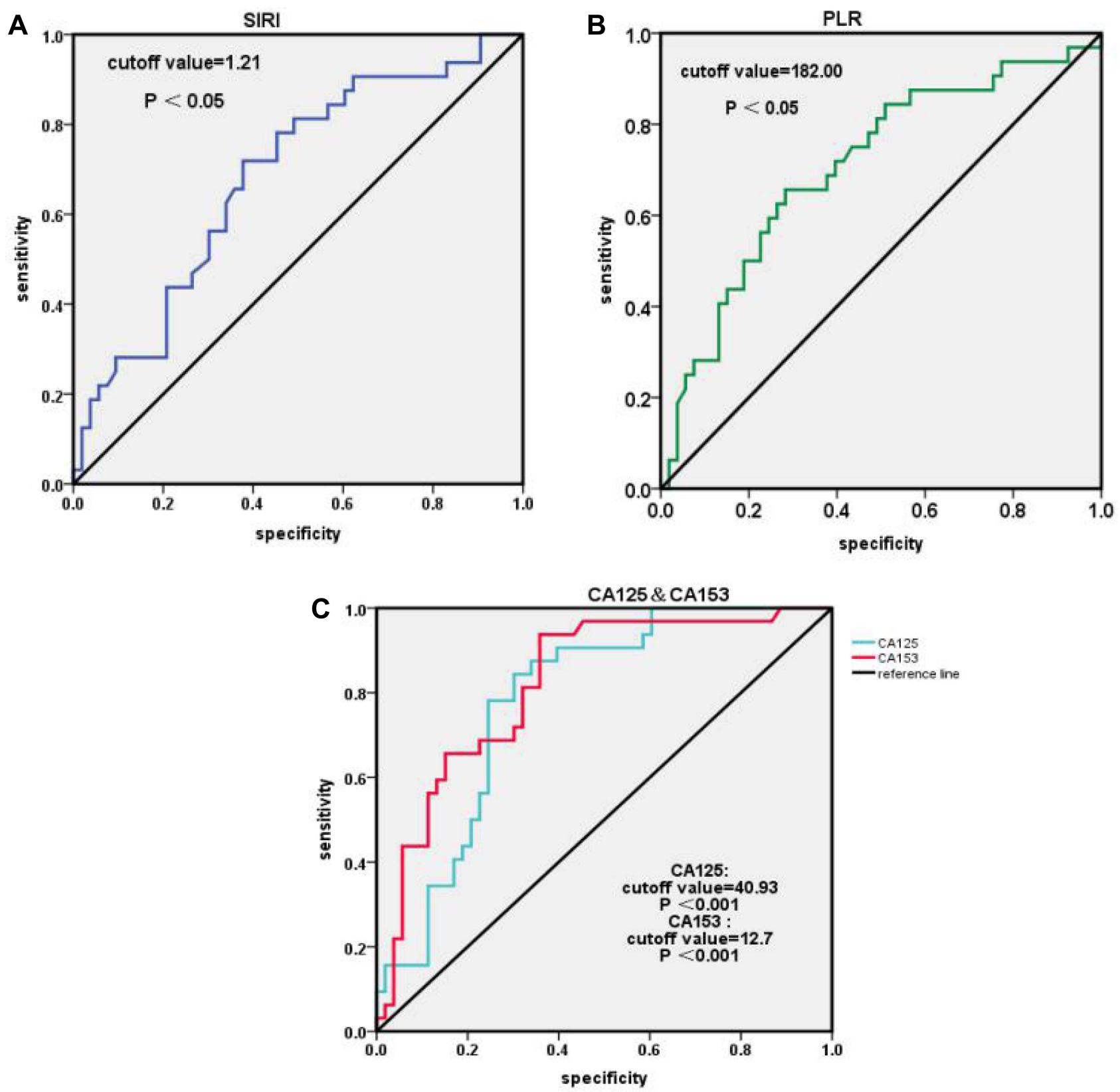

Figure 2 (A) ROC curve analysis for the association between SIRI with the high-stage ovarian malignant tumor. (B) ROC curve analysis for the association between PLR with the high-stage ovarian malignant tumor. (C) ROC curve analysis for the association between CAI25 and CAI53 with the high-stage ovarian malignant tumor. Notes: $P<0.05$ suggests significantly different. $P<0.00$ I suggest significantly different.

Abbreviations: SIRI, systemic inflammatory response index; PLR, platelet lymphocyte ratio; CAI25, cancer antigen I25; CAI53, cancer antigen I53.

ovarian malignancies and $100 \%$ of ovarian endometrioid carcinomas. ${ }^{16}$ HE4 is more sensitive than CA125 in the early diagnosis (stage I-II) of patients with ovarian malignancies. ${ }^{17}$ Studies have found that HE4 has higher sensitivity ( $90 \%$ and $83.3 \%)$ and specificity (95\% and $85 \%)$ in the diagnosis of epithelial ovarian malignancies than CA125, and the sensitivity of HE4 combined with CA125 can reach $96.7 \%{ }^{18}$ However, HE4 inspection is expensive and not suitable for routine inspection.
Ovarian tissue has been in various pro-inflammatory factors constantly, and the ovaries are damaged and repaired during repeated ovulation. Studies have shown that inflammation easily induces changes in the body's microenvironment, ${ }^{19}$ and the occurrence, development, invasion, metastasis and prognosis of tumors are accompanied by changes in the immune inflammatory response. ${ }^{20}$ Inflammatory cells include neutrophils, lymphocytes and platelets, etc., which are considered to be key factors in 
Table 3 Comparison of SIRI, PLR Correlation with CAI25, CAI53 in Ovarian Malignancies

\begin{tabular}{|l|l|c|c|}
\hline & & CAI25 & CAI53 \\
\hline SIRI & $r$ & $0.25 I^{*}$ & 0.199 \\
& $P$ & 0.021 & 0.067 \\
PLR & $r$ & $0.25 I^{*}$ & 0.187 \\
& $P$ & 0.020 & 0.086 \\
\hline
\end{tabular}

Notes: *Means that the correlation is significant at 0.05 .

Abbreviations: SIRI, systemic inflammatory response index; PLR, platelet lymphocyte ratio; CAI25, cancer antigen 125; CAI53, cancer antigen 153; r, correlation coefficient.

tumorigenesis. ${ }^{21}$ One report has showed that the lymphocyte mononuclear ratio (LMR) has diagnostic value for benign ovarian tumors and ovarian malignant tumors. ${ }^{22}$ SIRI value is the ratio of neutrophils $\times$ monocytes/lymphocytes, which can more stably reflect the condition of the disease than when the three are alone. ${ }^{23}$ At present, there are few reports on the application of SIRI in ovarian tumors. Platelets can affect the inflammatory response signal pathway and the biological behavior of white blood cells. ${ }^{24}$ Platelets can also promote the proliferation and metastasis of tumor cells. $^{25}$ PLR value is the ratio of platelets/lymphocytes. Two reports showed that PLR was one of the indicators of platelet activation status, and it could be used as a predictor of many cancers. ${ }^{26,27}$ The results of this study showed that the levels of SIRI, PLR in patients with ovarian malignant tumors were higher than that of ovarian benign tumors, and the differences were statistically significant. They were useful indicators of predicting benign and malignant ovarian tumors before surgery.

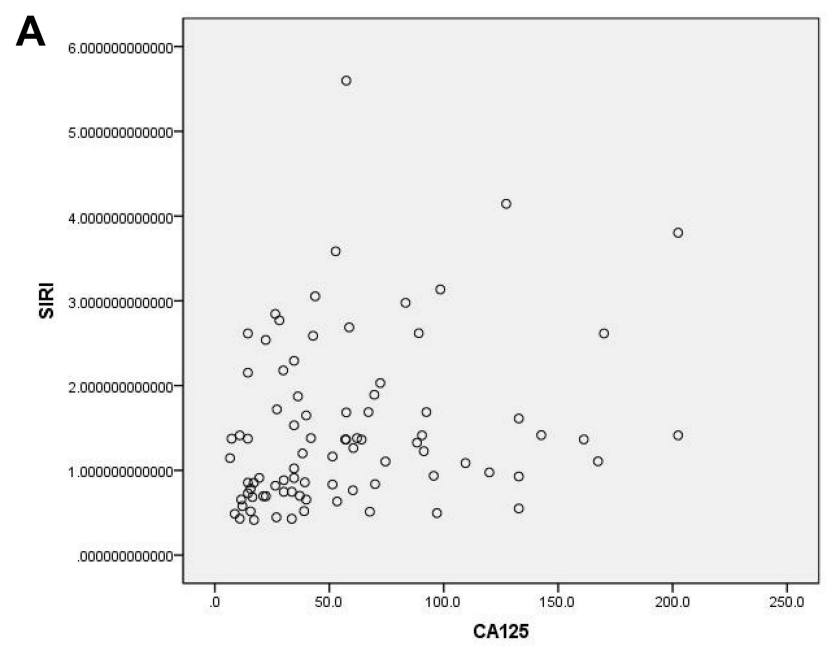

The ovarian malignant tumors are further divided into two groups: stage I-II is the low-stage ovarian malignant tumor group, and stage III-IV is the high-stage ovarian malignant tumor group. Studies have reported that the neutrophil to monocyte ratio (NLR) was related to the staging of epithelial ovarian cancer, and was associated with poor prognosis. ${ }^{28,29}$ SIRI is composed of the number of neutrophils, monocytes, and lymphocytes, which can be regarded as a comprehensive index of NLR and LMR. Another report showed that PLR had potential clinical value in predicting advanced stage disease or suboptimal surgery. ${ }^{30}$ Similar to previous studies, the results of this study show that SIRI and PLR levels were higher in the high stage group of ovarian malignant tumors than that in the low stage group. The ROC curve analysis showed that SIRI and PLR levels could be used to predict different stages of ovarian malignancies as CA125 and CA153.

Our study had some limitations. First, this is a retrospective study, which has a relatively small number of cases. In this study, nonsurgical patients were excluded from a control group. Thus, selection bias might have affected the study results. Second, healthy women are not included as a normal control group, we will do further research in the future work.

\section{Conclusion}

1. SIRI and PLR examinations were convenient and economical, and can be used as preliminary diagnostic indicators for ovarian malignant tumors and ovarian benign tumors.

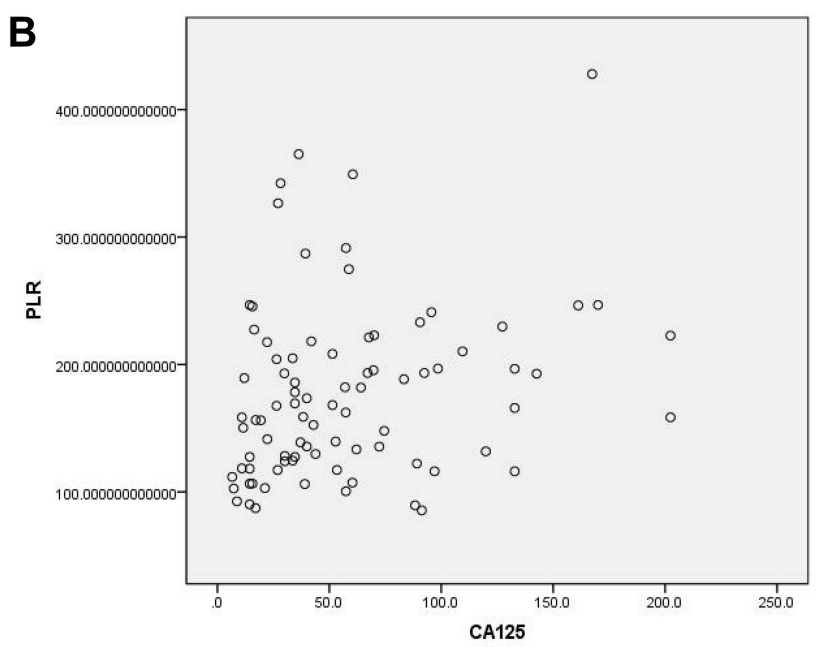

Figure 3 (A) Scatterplot of correlation analysis between SIRI and CAI25 in ovarian malignancies. (B) Scatterplot of correlation analysis between PLR and CAI25 in ovarian malignancies.

Note: $P<0.05$ suggests significantly different.

Abbreviations: SIRI, systemic inflammatory response index; CAI25, cancer antigen 125; PLR, platelet lymphocyte ratio. 
2. SIRI and PLR differentiated between low stage and high stage ovarian cancer.

3. SIRI and PLR were positively associated with CA125 for ovarian malignant tumor.

\section{Ethics}

This study was approved by the ethics committee of the Department of Gynecology of Fujian Maternity and Child Health Hospital, Affiliated Hospital of Fujian Medical University (the ethical approval number:2021KLRD09039. This article was a retrospective study, patient parental consent to review their medical records was not required by the ethics Committee of Fujian Maternity and Child Health Hospital, Affiliated Hospital of Fujian Medical University. This study strictly kept the patients' information confidential. The study complied with the Declaration of Helsinki.

\section{Acknowledgments}

The Authors would like to thank the Blood Transfusion Department, Fujian Maternity and Child Health Hospital, Affiliated Hospital of Fujian Medical University for helping to perform part of this work.

\section{Disclosure}

The authors report no conflicts of interest in this work.

\section{References}

1. Chen W, Zheng R, Baade PD, et al. Cancer statistics in China, 2015. CA Cancer J Clin. 2016;66(2):115-132. doi:10.3322/caac.21338

2. De Vorkin L, Hattersley M, Kim P, et al. Autophagy inhibition enhances sunitinib efficacy in clear cell ovarian carcinoma. Mol Cancer Res. 2017;15(3):250-258. doi:10.1158/1541-7786.MCR-160132

3. Torre LA, Trabert B, De Santis CE, et al. Ovarian cancer statistics, 2018. CA CANCER J CLIN. 2018;68:284-296. doi:10.3322/ caac. 21456

4. Wang YX, Wang YX, Li YK, et al. The emerging roles of extracellular vesicles in ovarian cancer. Curr Drug Metab. 2021;22(2):139-149. doi:10.2174/1389200221666201110155721

5. Akinwunmi BO, Babic A, Vitonis AF, et al. Chronic medical conditions and CA125 levels among women without ovarian cancer. Cancer Epidemiol Prev Biomarkers. 2018;27(12):1483-1490. doi:10.1158/ 1055-9965.EPI-18-0203

6. Feng F, Tian Y, Xu G, et al. Diagnostic and prognostic value of CEA, CA19-9, AFP and CA125 for early gastric cancer. BMC Cancer. 2017;17(1):1-6. doi:10.1186/s12885-017-3738-y

7. Fan HY, Duan DM, Liu YF. The diagnostic value of determination of serum GOLPH3 associated with CA125, CA19. 9 in patients with ovarian cancer. Eur Rev Med Pharmacol Sci. 2017;21(18):4039-4044.

8. Peng SJ, Wang CF, Yu YJ, et al. CYFRA21-1/TG ratio as an accurate risk factor to predict eye metastasis in nasopharyngeal carcinoma: a STROBE-compliant article. Medicine. 2020;99:46. doi:10.1097/ MD.0000000000022773
9. Stall KE, Martin EW Jr. Plasma carcinoembryonic antigen levels in ovarian cancer patients: a chart review and survey of published data. J Reprod Med. 1981;26(2):73-79.

10. Ness RB, Grisso JA, Cottreau C, et al. Factors related to inflammation of the ovarian epithelium and risk of ovarian cancer. Epidemiology. 2000;11(2):111-117. doi:10.1097/00001648200003000-00006

11. Abu-Shawer O, Abu-Shawer M, Haimour A, et al. Hematologic markers of distant metastases in gastric cancer. $J$ Gastrointest Oncol. 2019;10(3):529. doi:10.21037/jgo.2019.01.12

12. Mezouar S, Frère C, Darbousset R, et al. Role of platelets in cancer and cancer-associated thrombosis: experimental and clinical evidences. Thromb Res. 2016;139:65-76. doi:10.1016/j. thromres.2016.01.006

13. Lammert J, Skandarajah AR, Shackleton K, et al. Outcomes of women at high familial risk for breast cancer: an 8-year single-center experience. Asia Pac J Clin Oncol. 2020;16(2):27-37.

14. Morse CB, Norquist BM, Harrell MI, et al. Neoplastic cellularity is associated with clinical and molecular features of high-grade serous ovarian carcinoma. Gynecol Oncol. 2016;143(2):389-392. doi:10.1016/j.ygyno.2016.08.324

15. Dochez V, Caillon H, Vaucel E, Dimet J, Winer N, Ducarme G. Biomarkers and algorithms for diagnosis of ovarian cancer: CA125, HE4, RMI and ROMA, a review. J Ovarian Res. 2019;12(1):28. doi:10.1186/s13048-019-0503-7

16. Drapkin R, Von Horsten HH, Lin Y, et al. Human epididymis protein 4 (HE4) is a secreted glycoprotei $\mathrm{n}$ that is overexpressed by serous and endometrioid ovarian carci nomas. Cancer Res. 2005;65 (6):2162-2169. doi:10.1158/0008-5472.CAN-04-3924

17. Havrilesky LJ, Whitehead CM, Rubatt JM, et al. Evaluation of biomarker panels for early stage ovarian cancer detection and monitoring for disease recurrence. Gynecol Oncol. 2008;110 (3):374-382. doi:10.1016/j.ygyno.2008.04.041

18. Hamed EO, Ahmed H, Sedeek OB, Mohammed AM, Abd-Alla AA, Ghaffar HMA. Significance of HE4 estimation in comparison with CA125 in diagnosis of ovarian cancer and assessment of treatment response. Diagn Pathol. 2013;8(1):11-18. doi:10.1186/1746-1596-8-11

19. Rasmussen CB, Kjaer SK, Albieri V, et al. Pelvic information disease and the risk of ovarian cancer and borderline ovarian tumor: a pooled analysis of 13 case-control studies. Am J Epidemiol. 2017;185 (1):8-20. doi:10.1093/aje/kww161

20. Hanahan D, Weinberg RA. Hallmarks of cancer: the next generation. Cell. 2011;144(5):646-674. doi:10.1016/j.cell.2011.02.013

21. Gregory AD, Houghton AM. Tumor-associated neutrophils: new targets for cancer therapy. Cancer Res. 2011;71(7):2411-2416. doi:10.1158/0008-5472.CAN-10-2583

22. Qi Q, Zhuang L, Shen Y, et al. A novel systemic inflammation response index (SIRI) for predicting the survival of patients with pancreatic cancer after chemotherapy. Cancer. 2016;122 (14):2158-2167. doi:10.1002/cncr.30057

23. Yang B. The Clinical Significance of Preoperative LMR and PNI in the Diagnosis of Ovarian Malignant Tumors [D]. Yanbian University; 2019.

24. Jenne CN, Kubes P. Platelets in inflammation and infection. Platelets. 2015;26(4):286. doi:10.3109/09537104.2015.1010441

25. Davis A, Afsharkharghan V, Sood AK. Platelet effects on ovarian cancer. Semin Oncol. 2014;41(3):378-384. doi:10.1053/j. seminoncol.2014.04.004

26. Li QQ, Lu ZH, Yang L, et al. Neutrophil count and the inflammation-based Glasgow prognostic score predict survival in patients with advanced gastric cancer receiving first-line chemotherapy. Asian Pac J Cancer Prev. 2014;15(2):945-950. doi:10.7314/APJCP.2014.15.2.945

27. Shen J, Zhu Y, Wu W, et al. Prognostic role of neutrophil-tolymphocyte ratio in locally advanced rectal cancer treated with neoadjuvant chemoradiotherapy. Med Sci Monit. 2017;23:315-324. 
28. Yildiz Y, Kucukzeybek Y, Alacacioglu A, et al. Prognostic value of preoperative neutrophil-to-lymphocyte ratio and platelet-tolymphocyte ratio in patients with epithelial ovarian cancer. Eur Gynaecol Oncol. 2017;38(3):444-448.

29. Miao Y, Yan Q, Li S, et al. Neutrophil to lymphocyte ratio and platelet to lymphocyte ratio are predictive of chemotherapeutic response and prognosis in epithelial ovarian cancer patients treated with platinum-based chemotherapy. Cancer Biomarkers. 2016;17 (1):33-40. doi:10.3233/CBM-160614
30. Raungkaewmanee S, Tangjitgamol S, Manusirivithaya S, et al. Platelet to lymphocyte ratio as a prognostic factor for epithelial ovarian cancer. J Gynecol Oncol. 2012;23(4):265-273. doi:10.3802/ jgo.2012.23.4.265

\section{Publish your work in this journal}

The International Journal of General Medicine is an international, peer-reviewed open-access journal that focuses on general and internal medicine, pathogenesis, epidemiology, diagnosis, monitoring and treatment protocols. The journal is characterized by the rapid reporting of reviews, original research and clinical studies across all disease areas. The manuscript management system is completely online and includes a very quick and fair peer-review system, which is all easy to use. Visit http://www.dovepress.com/ testimonials.php to read real quotes from published authors. 